\title{
Existence and nonexistence of positive solutions for Dirichlet-type boundary value problem of nonlinear fractional differential equation
}

\author{
Xinyi Liu, Zhijun Zeng*
}

School of Mathematics and Statistics, Northeast Normal University, 5268 Renmin Street, Changchun, Jilin 130024, P. R. China.

Communicated by S. S. Chang

\begin{abstract}
In this paper, we investigate the existence and nonexistence of positive solutions for nonlinear fractional differential equation boundary value problem. By means of fixed-point theorems on a cone and the properties of Green function, some sufficient criteria are established. Our results can be considered as an extension of some previous results. (C)2017 all rights reserved.

Keywords: Positive solution, fractional differential equation, boundary value problem, the Krasnoselskii fixed point theorem. 2010 MSC: 93B05, 93C05, 93C55.
\end{abstract}

\section{Introduction}

Fractional calculus is a 300-year-old mathematical topic, starting from 30 September 1695, when the derivative of order $\alpha=\frac{1}{2}$ was described by Leibniz [1]. During the last few decades, fractional-order differential equations have been of great interest. The main advantage of fractional-order differential equations in comparison with classical integer-order ones is that fractional derivatives provide an excellent tool for the description of memory and hereditary properties of various processes. For an extensive collection of such results, we refer the readers to the monographs by Miller and Ross [8], Oldham and Spanier [9], and Poldubny [10].

Recently, there are some papers dealing with the existence and multiplicity of solutions of nonlinear initial value fractional differential equation by the use of techniques of nonlinear analysis $[2,3,5,6]$. In [3] and [6], the authors considered the Dirichlet-type boundary value problem for fractional differential equations

$$
\begin{aligned}
D_{0^{+}}^{\alpha} \mathrm{u}(\mathrm{t})+\mathrm{f}(\mathrm{t}, \mathrm{u}(\mathrm{t})) & =0, \quad 0<\mathrm{t}<1, \\
\mathrm{u}(0)=\mathrm{u}(1) & =0,
\end{aligned}
$$

where $1<\alpha \leqslant 2$ is a real number and $\mathrm{D}_{0^{+}}^{\alpha}$ is the standard Riemann-Liouville derivative, and $f:[0,1] \times$ $[0, \infty) \rightarrow[0, \infty)$ is continuous. By the use of techniques of fixed-point theorems on cone, the authors

\footnotetext{
*Corresponding author

Email addresses: liuxy394@nenu.edu.cn (Xinyi Liu), zthzzj@amss .ac.cn (Zhijun Zeng)
} 
discussed the existence and multiplicity of positive solutions for (1.1). Though some results have been obtained for system (1.1), such systems are not well studied yet. In this paper, motivated by the work of $[4,11,12]$, we proceed to develop more results for the existence and multiplicity of positive solutions of system (1.1). Moreover, we will discuss the nonexistence of positive solutions which is rarely discussed in previous work.

The tree of this paper is organized as follows. In Sections 2, we list some useful definitions and properties, and present the properties of Green Function of fractional differential equations with the boundary value problem. In Section 3, we establish some sufficient conditions for the existence of positive solutions for (1.1). Finally, in Section 4, we discuss the nonexistence of positive solutions of (1.1).

\section{Preliminaries}

In this section, we will present several foundational definitions of fractional calculus and preliminary results. For more details, one can see $[3,6]$.

Definition 2.1. The fractional integral of order $\alpha>0$ of a function $y:(0, \infty) \rightarrow \mathbb{R}$ is given by

$$
\mathrm{I}_{0^{+}}^{\alpha} \mathrm{y}(\mathrm{t})=\frac{1}{\Gamma(\alpha)} \int_{0}^{\mathrm{t}}(\mathrm{t}-\mathrm{s})^{\alpha-1} \mathrm{y}(\mathrm{s}) \mathrm{ds},
$$

provided that the right side is pointwise defined on $(0, \infty)$.

Definition 2.2. The fractional derivative of order $\alpha>0$ of a continuous function $y:(0, \infty) \rightarrow \mathbb{R}$ is given by

$$
D_{0^{+}}^{\alpha} y(t)=\frac{1}{\Gamma(n-\alpha)}\left(\frac{d}{d t}\right)^{n} \int_{0}^{t} \frac{y(s)}{(t-s)^{\alpha-n+1}} d s,
$$

where $n=[\alpha]+1$, provided that the right side is pointwise defined on $(0, \infty)$.

Lemma 2.3. Let $\alpha>0$ and $u \in \mathrm{C}(0,1) \cap \mathrm{L}(0,1)$, then the fractional differential equation

$$
\mathrm{D}_{0^{+}}^{\alpha} \mathrm{u}(\mathrm{t})=0
$$

has solutions

$$
\mathrm{u}(\mathrm{t})=\mathrm{c}_{1} \mathrm{t}^{\alpha-1}+\mathrm{c}_{2} \mathrm{t}^{\alpha-2}+\cdots+\mathrm{c}_{\mathrm{n}} \mathrm{t}^{\alpha-\mathrm{n}},
$$

for some $c_{i} \in \mathbb{R}, i=1,2, \cdots, n$, and $n$ is the smallest integer greater than or equal to $\alpha$.

Lemma 2.4. Assume that $\mathrm{u} \in \mathrm{C}(0,1) \cap \mathrm{L}(0,1)$ with a fractional derivative of order $\alpha>0$. Then

$$
\mathrm{I}_{0^{+}}^{\alpha} \mathrm{D}_{0^{+}}^{\alpha} \mathrm{u}(\mathrm{t})=\mathrm{u}(\mathrm{t})+\mathrm{C}_{1} \mathrm{t}^{\alpha-1}+\mathrm{C}_{2} \mathrm{t}^{\alpha-2}+\cdots+\mathrm{C}_{\mathrm{n}} \mathrm{t}^{\alpha-\mathrm{n}},
$$

for some $c_{i} \in \mathbb{R}, i=1,2, \cdots, n, n$ is the smallest integer greater than or equal to $\alpha$.

Lemma 2.5. Given $\mathrm{y} \in \mathrm{C}[0,1]$ and $1<\alpha \leqslant 2$, the unique solution of

$$
\begin{aligned}
D_{0^{+}}^{\alpha} \mathrm{u}(\mathrm{t})+\mathrm{y}(\mathrm{t}) & =0, \quad 0<\mathrm{t}<1, \\
\mathrm{u}(0)=\mathfrak{u}(1) & =0
\end{aligned}
$$

is

$$
u(t)=\int_{0}^{1} G(t, s) y(s) d s
$$

where

$$
G(t, s)= \begin{cases}\frac{[t(1-s)]^{\alpha-1}-(t-s)^{\alpha-1}}{\Gamma(\alpha)}, & 0 \leqslant s \leqslant t \leqslant 1 \\ \frac{[t(1-s)]^{\alpha-1}}{\Gamma(\alpha)}, & 0 \leqslant t \leqslant s \leqslant 1 .\end{cases}
$$


Lemma 2.6. $u(t)$ is a solution of (1.1), if and only if it is also a solution of the following integral equation

$$
u(t)=\int_{0}^{1} G(t, s) f(s, u(s)) d s,
$$

where $\mathrm{G}(\mathrm{t}, \mathrm{s})$ is defined in $(2.1)$.

Lemma 2.7. Let $\mathrm{G}^{*}(\mathrm{t}, \mathrm{s}):=\mathrm{t}^{2-\alpha} \mathrm{G}(\mathrm{t}, \mathrm{s})$, then

$$
\frac{\alpha-1}{\Gamma(\alpha)} \mathrm{t}(1-\mathrm{t}) \mathrm{s}(1-\mathrm{s})^{\alpha-1} \leqslant \mathrm{G}^{*}(\mathrm{t}, \mathrm{s}) \leqslant \frac{1}{\Gamma(\alpha)} \mathrm{s}(1-\mathrm{s})^{\alpha-1},
$$

for $\mathrm{t}, \mathrm{s} \in(0,1)$.

Next, we give the definition for cone and the famous fixed point theorem that will be needed in our arguments [7].

Definition 2.8. Let $X$ be a Banach space and $E$ be a closed nonempty subset of $X$. $E$ is said to be a cone if

(i) $\alpha u+\beta v \in E$ for all $u, v \in E$ and all $\alpha, \beta>0$;

(ii) $\mathfrak{u},-\mathfrak{u} \in$ E imply $\mathfrak{u}=0$.

Theorem 2.9. Let $\mathrm{X}$ be a Banach space, and let $\mathrm{E} \subset \mathrm{X}$ be a cone in $\mathrm{X}$. Assume $\Omega_{1}, \Omega_{2}$ are open subsets of $\mathrm{X}$ with $0 \in \Omega_{1}, \bar{\Omega}_{1} \subset \Omega_{2}$ and let $\mathrm{T}: \mathrm{E} \cap\left(\bar{\Omega}_{2} \backslash \Omega_{1}\right) \longrightarrow \mathrm{E}$ be a completely continuous operator such that either

(1) $\|\mathrm{T} y\| \geqslant\|\mathrm{y}\|$ for any $\mathrm{y} \in \mathrm{E} \cap \partial \Omega_{1}$, and $\|\mathrm{T} y\| \leqslant\|\mathrm{y}\|$ for any $\mathrm{y} \in \mathrm{E} \cap \partial \Omega_{2}$, or

(2) $\|\mathrm{T} y\| \leqslant\|y\|$ for any $\mathrm{y} \in \mathrm{E} \cap \partial \Omega_{1}$, and $\|\mathrm{T} y\| \geqslant\|\mathrm{y}\|$ for any $\mathrm{y} \in \mathrm{E} \cap \partial \Omega_{2}$.

Then $\mathrm{T}$ has a fixed point in $\mathrm{E} \cap\left(\bar{\Omega}_{2} \backslash \Omega_{1}\right)$.

\section{Existence of positive solutions of (1.1)}

In this section, we establish the positive solutions of (1.1) by applying the fixed point theorems on a cone. In order to explore the existence of positive solutions of (1.1), we suppose the following hypotheses are always satisfied in the sequel.

(A) $f(t, u)$ is continuous on $[0,1] \times[0, \infty)$, and there exist $g \in C([0,+\infty),[0,+\infty)), q_{1}, q_{2} \in C((0,+\infty),(0$, $+\infty)$ ) such that

$$
q_{1}(t) g(y) \leqslant f\left(t, t^{\alpha-2} y\right) \leqslant q_{2}(t) g(y), t \in(0,1), y \in[0, \infty),
$$

where

$$
\int_{0}^{1} \mathrm{q}_{i}(\mathrm{~s}) \mathrm{d} \mathrm{s}<+\infty, i=1,2
$$

Let $E=C[0,1]$ be endowed with the ordering $u \leqslant v$ if $u(t) \leqslant v(t)$ for all $t \in[0,1]$, and the maximum norm $\|\mathrm{u}\|=\max _{0 \leqslant \mathrm{t} \leqslant 1}|\mathrm{u}(\mathrm{t})|$.

Define the cone $P \subset E$ by $P=\{u \in E \mid u(t) \geqslant 0\}$. Then we have the following lemma.

Lemma 3.1. Let $\mathrm{T}: \mathrm{P} \rightarrow \mathrm{E}$ be the operator defined by

$$
(T u)(t)=\int_{0}^{1} G(t, s) f(s, u(s)) d s
$$

then $\mathrm{T}: \mathrm{P} \rightarrow \mathrm{P}$ is completely continuous. 
It is clear that $u(t)$ is a positive solution of (1.1), whenever $u(t)$ is a fixed point of $T$, namely, $u(t)=$ $(\mathrm{Tu})(\mathrm{t})$.

Define the cone $\mathrm{K} \subset \mathrm{E}$ by

$$
K=\{y \in E \mid y(t) \geqslant(\alpha-1) t(1-t)\|y\|\},
$$

and an operator $\mathrm{T}^{*}: \mathrm{K} \rightarrow \mathrm{E}$ as follows

$$
\left(T^{*} y\right)(t)=\int_{0}^{1} G^{*}(t, s) f\left(s, s^{\alpha-2} y(s)\right) d s
$$

Then one has the following lemma.

Lemma 3.2. $\mathrm{T}^{*}(\mathrm{~K}) \subset \mathrm{K}$ and $\mathrm{T}^{*}: \mathrm{K} \rightarrow \mathrm{K}$ is completely continuous.

For the sake of convenience and simplicity, we introduce the following notations

$$
\begin{aligned}
g^{0} & =\lim _{y \rightarrow 0} \max \frac{g(y)}{y}, & g^{\infty} & =\lim _{y \rightarrow \infty} \max \frac{g(y)}{y}, \\
g_{0} & =\lim _{y \rightarrow 0} \min \frac{g(y)}{y}, & g_{\infty} & =\lim _{y \rightarrow \infty} \min \frac{g(y)}{y} .
\end{aligned}
$$

Moreover, define, for $r$ a positive number, $\Omega_{r}$ by

$$
\Omega_{\mathrm{r}}=\{\mathrm{y} \in \mathrm{C}[0,1] \mid\|y\|<\mathrm{r}\} .
$$

Note that $\partial \Omega_{r}=\{y \in C[0,1] \mid\|y\|=r\}$.

Our first result is as follows:

Theorem 3.3. Assume that

$$
\text { (P1) } g_{0}=\infty \text { and }(\mathrm{P} 2) \mathrm{g}^{\infty}=0
$$

hold. Then system (1.1) has at least one positive solution.

Proof. First, in view of $g_{0}=\infty$, there exist $\rho_{0}>0, M>0$ satisfying

$$
\frac{M(\alpha-1)^{2}}{4 \Gamma(\alpha)} \int_{0}^{1} s^{2}(1-s)^{\alpha} q_{1}(s) d s \geqslant 1
$$

such that $\mathrm{g}(\mathrm{y}) \geqslant M y$ for $0<y \leqslant \rho_{0}$. Then, for any $y \in \Omega_{\rho_{0}}, 0<y<\rho_{0}$, by Lemma 2.7 and (3.1), we have

$$
\begin{aligned}
\left\|T^{*} y\right\| & \geqslant\left(T^{*} y\right)\left(\frac{1}{2}\right) \\
& =\int_{0}^{1} G^{*}\left(\frac{1}{2}, s\right) f\left(s, s^{\alpha-2} y(s)\right) d s \\
& \geqslant \frac{\alpha-1}{4 \Gamma(\alpha)} \int_{0}^{1} s(1-s)^{\alpha-1} q_{1}(s) g(y(s)) d s \\
& \geqslant \frac{M(\alpha-1)}{4 \Gamma(\alpha)} \int_{0}^{1} s(1-s)^{\alpha-1} q_{1}(s) y(s) d s \\
& \geqslant \frac{M(\alpha-1)^{2}}{4 \Gamma(\alpha)}\|y\| \int_{0}^{1} s^{2}(1-s)^{\alpha} q_{1}(s) d s \\
& \geqslant\|y\|,
\end{aligned}
$$

which implies that $\left\|T^{*} y\right\| \geqslant\|y\|$ for any $y \in K \cap \partial \Omega_{\rho_{0}}$. 
On the other hand, by using $g^{\infty}=0$, there exist $M^{*}>\rho_{0}>0$ and $\varepsilon>0$ satisfying

$$
\frac{\varepsilon}{\Gamma(\alpha)} \int_{0}^{1} s(1-s)^{\alpha-1} \mathrm{q}_{2}(\mathrm{~s}) \mathrm{d} s \leqslant \frac{1}{2}
$$

such that $g(y) \leqslant \varepsilon y$ for $y>M^{*}$.

Take

$$
\rho_{1}>M^{*}+\frac{1}{\varepsilon} \max _{y \in\left[0, M^{*}\right]}\{g(y)\} .
$$

Then by Lemma 2.7 and (3.1), we have

$$
\begin{aligned}
\left(T^{*} y\right)(t) & \leqslant \frac{1}{\Gamma(\alpha)} \int_{0}^{1} s(1-s)^{\alpha-1} f\left(s, s^{\alpha-2} y(s)\right) d s \\
& \leqslant \frac{1}{\Gamma(\alpha)} \int_{0}^{1} s(1-s)^{\alpha-1} q_{2}(s) g(y(s)) d s \\
& \leqslant \frac{1}{\Gamma(\alpha)}\left\{\int_{I_{1}} s(1-s)^{\alpha-1} q_{2}(s) \max \{g(y(s))\} d s+\int_{I_{2}} s(1-s)^{\alpha-1} q_{2}(s) \varepsilon y(s) d s\right\} \\
& \leqslant \frac{1}{\Gamma(\alpha)} \int_{0}^{1} s(1-s)^{\alpha-1} q_{2}(s) d s \max _{y(s) \in \mathrm{I}_{1}}\{g(y(s))\}+\frac{\varepsilon}{\Gamma(\alpha)}\|y\| \int_{0}^{1} s(1-s)^{\alpha-1} q_{2}(s) d s \\
& \leqslant \frac{\rho_{1}}{2}+\frac{\|y\|}{2} \\
& =\|y\|,
\end{aligned}
$$

where $I_{1}=\left\{s \in[0,1] \mid 0 \leqslant y(s) \leqslant M^{*}\right\}, I_{2}=\left\{s \in[0,1] \mid y(s)>M^{*}\right\}$. This implies that $\left\|T^{*} y\right\| \leqslant\|y\|$ for any $y \in K \cap \partial \Omega_{\rho_{1}}$.

Thus, by Theorem $2.9, T^{*}$ has a fixed point $y$ in $K \cap\left(\bar{\Omega}_{\rho_{1}} \backslash \Omega_{\rho_{0}}\right)$, that is, $y(t)=\int_{0}^{1} G^{*}(t, s) f\left(s, s^{\alpha-2} y(s)\right) d s$, $t \in[0,1]$. It is obvious that $u(t)=t^{\alpha-2} y(t)$ is a fixed point of $T$, and it satisfies $u(t)=\int_{0}^{1} G(t, s) f(s, u(s)) d s$, $\mathrm{t} \in[0,1]$. Finally, we prove $u(0)=\mathfrak{u}(1)=0$.

From $y \in C[0,1]$ and $(\mathrm{A})$, we have

$$
\begin{aligned}
\lim _{t \rightarrow 0} u(t) & =\lim _{t \rightarrow 0} \int_{0}^{1} G(t, s) f(s, u(s)) d s \\
& =\lim _{t \rightarrow 0} \int_{0}^{1} G(t, s) f\left(s, s^{\alpha-2} y(s)\right) d s \\
& \leqslant \lim _{t \rightarrow 0} \int_{0}^{1} G(t, s) q_{2}(s) g(y(s)) d s \\
& \leqslant \lim _{t \rightarrow 0} \int_{0}^{1} G(t, s) q_{2}(s) d s \max _{0 \leqslant\|y\| \leqslant \rho_{1}} g(y(s)) \\
& =0 .
\end{aligned}
$$

So, $u(0)=0$. By $(2.1)$, it is easy to see that $u(1)=y(1)=0$. Hence, system (1.1) has a positive solution $u(t)=t^{\alpha-2} y(t)$. Therefore the proof is completed.

Theorem 3.4. Assume that

$$
\text { (P3) } g^{0}=0 \text { and }\left(\text { P4) } g_{\infty}=\infty\right.
$$

hold. Then system (1.1) has at least one positive solution.

Proof. Since $\mathrm{g}^{0}=0$, for any $\varepsilon>0$ satisfying

$$
\frac{\varepsilon}{\Gamma(\alpha)} \int_{0}^{1} \mathrm{~s}(1-\mathrm{s})^{\alpha-1} \mathrm{q}_{2}(\mathrm{~s}) \mathrm{d} \mathrm{s} \leqslant 1,
$$

there exists $\rho_{2}>0$ such that $g(y) \leqslant \varepsilon y$ for $y \leqslant \rho_{2}$. 
Then by Lemma 2.7 and (3.1), we get

$$
\begin{aligned}
\left(T^{*} y\right)(t) & \leqslant \frac{1}{\Gamma(\alpha)} \int_{0}^{1} s(1-s)^{\alpha-1} f\left(s, s^{\alpha-2} y(s)\right) d s \\
& \leqslant \frac{1}{\Gamma(\alpha)} \int_{0}^{1} s(1-s)^{\alpha-1} q_{2}(s) g(y(s)) d s \\
& \leqslant \frac{\varepsilon}{\Gamma(\alpha)} \int_{0}^{1} s(1-s)^{\alpha-1} q_{2}(s) y(s) d s \\
& \leqslant \frac{\varepsilon}{\Gamma(\alpha)}\|y\| \int_{0}^{1} s(1-s)^{\alpha-1} q_{2}(s) d s \\
& \leqslant\|y\|,
\end{aligned}
$$

which shows that $\left\|T^{*} y\right\| \leqslant\|y\|$ for $y \in K \cap \partial \Omega_{\rho_{2}}$.

Further, since $g_{\infty}=\infty$, for any $M_{1}>0$ satisfying

$$
\frac{3 M_{1}(\alpha-1)^{2}}{64 \Gamma(\alpha)} \int_{\frac{1}{4}}^{\frac{3}{4}} s(1-s)^{\alpha-1} q_{1}(s) d s \geqslant 1,
$$

there exists $\rho_{3}^{*}>\rho_{2}>0$ such that $g(y)>M_{1} y$ for $y \geqslant \rho_{3}^{*}$. Let

$$
\rho_{3}=\frac{16 \rho_{3}^{*}}{3(\alpha-1)}+\rho_{2} \text {. }
$$

Then for any $y \in K \cap \partial \Omega_{\rho_{3}}$, we have

$$
y(t) \geqslant \frac{3(\alpha-1)}{16}\|y\|=\frac{3(\alpha-1)}{16} \rho_{3}>\rho_{3}^{*}, \quad t \in\left[\frac{1}{4}, \frac{3}{4}\right] .
$$

Consequently,

$$
\begin{aligned}
\left\|T^{*} y\right\| & \geqslant\left(T^{*} y\right)\left(\frac{1}{2}\right) \\
& =\int_{0}^{1} G^{*}\left(\frac{1}{2}, s\right) f\left(s, s^{\alpha-2} y(s)\right) d s \\
& \geqslant \frac{\alpha-1}{4 \Gamma(\alpha)} \int_{0}^{1} s(1-s)^{\alpha-1} q_{1}(s) g(y(s)) d s \\
& \geqslant \frac{M_{1}(\alpha-1)}{4 \Gamma(\alpha)} \int_{0}^{1} s(1-s)^{\alpha-1} q_{1}(s) y(s) d s \\
& \geqslant \frac{3 M_{1}(\alpha-1)^{2}}{64 \Gamma(\alpha)}\|y\| \int_{\frac{1}{4}}^{\frac{3}{4}} s(1-s)^{\alpha-1} q_{1}(s) d s \\
& \geqslant\|y\|,
\end{aligned}
$$

which implies that $\left\|T^{*} y\right\| \geqslant\|y\|$ for any $y \in K \cap \partial \Omega_{\rho_{3}}$.

Therefore $T^{*}$ has a fixed point $y(t)$ in $K \cap\left(\bar{\Omega}_{\rho_{3}} \backslash \Omega_{\rho_{2}}\right)$. Clearly, system (1.1) has a positive solution $u(t)=t^{\alpha-2} y(t)$. So the proof is completed.

Theorem 3.5. Assume that the following two conditions hold:

(P5) there exists an $r_{1}>0$ such that $g(y) \geqslant M_{1} r_{1}$ for $\frac{3(\alpha-1)}{16} r_{1} \leqslant y \leqslant r_{1}$;

(P6) there exists an $r_{2}>0$ such that $g(y) \leqslant M_{2} r_{2}$ for $0<y \leqslant r_{2}$, 
where

$$
\begin{aligned}
& M_{1}=\left[\frac{\alpha-1}{4 \Gamma(\alpha)} \int_{\frac{1}{4}}^{\frac{3}{4}} s(1-s)^{\alpha-1} \mathrm{q}_{1}(s) \mathrm{d} s\right]^{-1}, \\
& M_{2}=\left[\frac{1}{\Gamma(\alpha)} \int_{0}^{1} s(1-s)^{\alpha-1} \mathrm{q}_{2}(s) \mathrm{d} s\right]^{-1} .
\end{aligned}
$$

Then system (1.1) has at least one positive solution.

Proof. Without loss of generality, we can assume that $r_{1}>r_{2}$. By (P5), Lemma 2.7, and (3.1), for any $\mathrm{y} \in \mathrm{K} \cap \partial \Omega_{\mathrm{r}_{1}}$, we have

$$
\begin{aligned}
\left\|T^{*} y\right\| \geqslant\left(T^{*} y\right)\left(\frac{1}{2}\right) & =\int_{0}^{1} G^{*}\left(\frac{1}{2}, s\right) f\left(s, s^{\alpha-2} y(s)\right) d s \\
& \geqslant \frac{\alpha-1}{4 \Gamma(\alpha)} \int_{\frac{1}{4}}^{\frac{3}{4}} s(1-s)^{\alpha-1} q_{1}(s) g(y(s)) d s \\
& \geqslant \frac{M_{1} r_{1}(\alpha-1)}{4 \Gamma(\alpha)} \int_{\frac{1}{4}}^{\frac{3}{4}} s(1-s)^{\alpha-1} q_{1}(s) d s \\
& =r_{1}=\|y\|,
\end{aligned}
$$

which leads to $\left\|T^{*} y\right\| \geqslant\|y\|$ for any $y \in K \cap \partial \Omega_{r_{1}}$.

On the other hand, by (P6), one has

$$
\begin{aligned}
\left(T^{*} y\right)(t) & \leqslant \frac{1}{\Gamma(\alpha)} \int_{0}^{1} s(1-s)^{\alpha-1} f\left(s, s^{\alpha-2} y(s)\right) d s \\
& \leqslant \frac{1}{\Gamma(\alpha)} \int_{0}^{1} s(1-s)^{\alpha-1} q_{2}(s) g(y(s)) d s \\
& \leqslant \frac{M_{2} r_{2}}{\Gamma(\alpha)} \int_{0}^{1} s(1-s)^{\alpha-1} q_{2}(s) d s \\
& =r_{2}=\|y\|,
\end{aligned}
$$

which yields $\left\|T^{*} y\right\| \leqslant\|y\|$ for any $y \in K \cap \partial \Omega_{r_{2}}$.

Therefore, from Theorem 2.9, $\mathrm{T}^{*}$ has a fixed point in $\mathrm{K} \cap\left(\overline{\Omega_{\mathrm{r}_{1}}} \backslash \Omega_{\mathrm{r}_{2}}\right)$. Further, we can obtain that system (1.1) has at least one positive solution. The proof is completed.

Theorem 3.6. Assume that (P2), (P3), and (P5) hold. Then system (1.1) has at least two positive solutions.

Proof. Firstly, by (P3), for any $\varepsilon>0$ satisfying

$$
\frac{\varepsilon}{\Gamma(\alpha)} \int_{0}^{1} s(1-s)^{\alpha-1} q_{2}(s) d s \leqslant 1,
$$

there exists $\rho_{4} \in\left(0, r_{1}\right)$ such that $g(y) \leqslant \varepsilon y$ for $y \leqslant \rho_{4}$.

Then by Lemma 2.7 and (3.1), we have

$$
\begin{aligned}
\left(T^{*} y\right)(t) & \leqslant \frac{1}{\Gamma(\alpha)} \int_{0}^{1} s(1-s)^{\alpha-1} f\left(s, s^{\alpha-2} y(s)\right) d s \\
& \leqslant \frac{1}{\Gamma(\alpha)} \int_{0}^{1} s(1-s)^{\alpha-1} q_{2}(s) g(y(s)) d s
\end{aligned}
$$




$$
\begin{aligned}
& \leqslant \frac{\varepsilon}{\Gamma(\alpha)} \int_{0}^{1} s(1-s)^{\alpha-1} \mathrm{q}_{2}(\mathrm{~s}) \mathrm{y}(\mathrm{s}) \mathrm{d} s \\
& \leqslant\|y\|,
\end{aligned}
$$

which shows that $\left\|T^{*} y\right\| \leqslant\|y\|$ for $y \in K \cap \partial \Omega_{\rho_{4}}$.

Secondly, in view of (P2), for any $\varepsilon>0$ satisfying

$$
\frac{\varepsilon}{\Gamma(\alpha)} \int_{0}^{1} s(1-s)^{\alpha-1} \mathrm{q}_{2}(\mathrm{~s}) \mathrm{d} s \leqslant \frac{1}{2}
$$

there exists an $M_{2}>0$, such that $g(y) \leqslant \varepsilon y$ for $y>M_{2}$. Let

$$
\rho_{5}>M_{2}+r_{1}+\frac{1}{\varepsilon} \max _{y \in\left[0, M_{2}\right]}\{g(y)\} .
$$

Then we have

$$
\begin{aligned}
\left(T^{*} y\right)(t) & \leqslant \frac{1}{\Gamma(\alpha)} \int_{0}^{1} s(1-s)^{\alpha-1} f\left(s, s^{\alpha-2} y(s)\right) d s \\
& \leqslant \frac{1}{\Gamma(\alpha)} \int_{0}^{1} s(1-s)^{\alpha-1} q_{2}(s) g(y(s)) d s \\
& \leqslant \frac{1}{\Gamma(\alpha)}\left\{\int_{I_{1}} s(1-s)^{\alpha-1} q_{2}(s) \max \{g(y(s))\} d s+\int_{I_{2}} s(1-s)^{\alpha-1} q_{2}(s) \varepsilon y(s) d s\right\} \\
& \leqslant \frac{1}{\Gamma(\alpha)} \int_{0}^{1} s(1-s)^{\alpha-1} q_{2}(s) \max \{g(y(s))\} d s+\frac{\varepsilon}{\Gamma(\alpha)}\|y\| \int_{0}^{1} s(1-s)^{\alpha-1} q_{2}(s) d s \\
& \leqslant \frac{1}{2 \varepsilon} \varepsilon \rho_{5}+\frac{\|y\|}{2} \\
& =\|y\|,
\end{aligned}
$$

where $I_{1}=\left\{s \in[0,1] \mid 0 \leqslant y(s) \leqslant M_{2}\right\}, \quad I_{2}=\left\{s \in[0,1] \mid y(s)>M_{2}\right\}$. Hence, $\left\|T^{*} y\right\| \leqslant\|y\|$ for $\mathrm{y} \in \mathrm{K} \cap \partial \Omega_{\rho_{5}}$.

Finally, set $\Omega_{r_{1}}=\left\{y \in C[0,1] \mid\|y\|<r_{1}\right\}$. Then, by (P5), we get

$$
\begin{aligned}
\left\|T^{*} y\right\| & \geqslant\left(T^{*} y\right)\left(\frac{1}{2}\right) \\
& =\int_{0}^{1} G^{*}\left(\frac{1}{2}, s\right) f\left(s, s^{\alpha-2} y(s)\right) d s \\
& \geqslant \frac{\alpha-1}{4 \Gamma(\alpha)} \int_{\frac{1}{4}}^{\frac{3}{4}} s(1-s)^{\alpha-1} q_{1}(s) g(y(s)) d s \\
& \geqslant \frac{M_{1}(\alpha-1)}{4 \Gamma(\alpha)} r_{1} \int_{\frac{1}{4}}^{\frac{3}{4}} s(1-s)^{\alpha-1} q_{1}(s) d s \\
& =r_{1}=\|y\|,
\end{aligned}
$$

which yields $\left\|T^{*} y\right\| \geqslant\|y\|$ for any $y \in K \cap \partial \Omega_{r_{1}}$.

Therefore, $T^{*}$ has a fixed point $y_{1}$ in $\bar{\Omega}_{r_{1}} \backslash \Omega_{\rho_{4}}$, and a fixed point $y_{2}$ in $\bar{\Omega}_{\rho_{5}} \backslash \Omega_{r_{1}}$. One can easily see that both $u_{1}=t^{\alpha-2} y_{1}$ and $u_{2}=t^{\alpha-2} y_{2}, t \in[0,1]$ are positive solutions of system (1.1). The proof is completed.

Theorem 3.7. Assume that (P1), (P4), and (P6) hold. Then system (1.1) has at least two positive solutions. 
Proof. In view of (P1), for any $\mathrm{M}_{3}>0$ satisfying

$$
\frac{M_{3}(\alpha-1)^{2}}{4 \Gamma(\alpha)} \int_{0}^{1} s^{2}(1-s)^{\alpha} q_{1}(s) d s \geqslant 1,
$$

there exists a $\rho_{6} \in\left(0, r_{2}\right)$ such that $g(y) \geqslant M_{3} y$ for $y \leqslant \rho_{6}$. Then by Lemma 2.7 and (3.1), we obtain

$$
\begin{aligned}
\left\|T^{*} y\right\| & \geqslant\left(T^{*} y\right)\left(\frac{1}{2}\right) \\
& =\int_{0}^{1} G^{*}\left(\frac{1}{2}, s\right) f\left(s, s^{\alpha-2} y(s)\right) d s \\
& \geqslant \frac{\alpha-1}{4 \Gamma(\alpha)} \int_{0}^{1} s(1-s)^{\alpha-1} q_{1}(s) g(y(s)) d s \\
& \geqslant \frac{M_{3}(\alpha-1)}{4 \Gamma(\alpha)} \int_{0}^{1} s(1-s)^{\alpha-1} q_{1}(s) y(s) d s \\
& \geqslant \frac{M_{3}(\alpha-1)^{2}}{4 \Gamma(\alpha)}\|y\| \int_{0}^{1} s^{2}(1-s)^{\alpha} q_{1}(s) d s \\
& \geqslant\|y\|,
\end{aligned}
$$

which yields $\left\|T^{*} y\right\| \geqslant\|y\|$ for any $y \in K \cap \partial \Omega_{\rho_{6}}$. In addition, by (P4), for any $M_{4}>0$ satisfying

$$
\frac{3 M_{4}(\alpha-1)^{2}}{64 \Gamma(\alpha)} \int_{\frac{1}{4}}^{\frac{3}{4}} s(1-s)^{\alpha-1} q_{1}(s) d s \geqslant 1,
$$

there exists $\rho_{7}^{*}>0$ such that $g(y)>M_{4} y$ for $y \geqslant \rho_{7}^{*}$. Take

$$
\rho_{7}=\frac{16 \rho_{7}^{*}}{3(\alpha-1)}+\rho_{2}
$$

then for any $y \in K \cap \partial \Omega_{\rho_{7}}$, we have

$$
y(t) \geqslant \frac{3(\alpha-1)}{16}\|y\|=\frac{3(\alpha-1)}{16} \rho_{7}>\rho_{7}^{*}, \quad t \in\left[\frac{1}{4}, \frac{3}{4}\right] .
$$

Furthermore,

$$
\begin{aligned}
\left\|T^{*} y\right\| & \geqslant\left(T^{*} y\right)\left(\frac{1}{2}\right) \\
& \geqslant \frac{\alpha-1}{4 \Gamma(\alpha)} \int_{0}^{1} s(1-s)^{\alpha-1} q_{1}(s) g(y(s)) d s \\
& \geqslant \frac{\alpha-1}{4 \Gamma(\alpha)} \int_{\frac{1}{4}}^{\frac{3}{4}} s(1-s)^{\alpha-1} q_{1}(s) g(y(s)) d s \\
& \geqslant \frac{M_{4}(\alpha-1)}{4 \Gamma(\alpha)} \int_{\frac{1}{4}}^{\frac{3}{4}} s(1-s)^{\alpha-1} q_{1}(s) y(s) d s \\
& \geqslant \frac{3 M_{4}(\alpha-1)^{2}}{64 \Gamma(\alpha)}\|y\| \int_{\frac{1}{4}}^{\frac{3}{4}} s(1-s)^{\alpha-1} q_{1}(s) d s \\
& \geqslant\|y\|,
\end{aligned}
$$

which implies that $\left\|T^{*} y\right\| \geqslant\|y\|$ for any $y \in K \cap \partial \Omega_{\rho_{7}}$. 
Finally, by (P6), we have

$$
\begin{aligned}
\left(T^{*} y\right)(t) & \leqslant \frac{1}{\Gamma(\alpha)} \int_{0}^{1} s(1-s)^{\alpha-1} f\left(s, s^{\alpha-2} y(s)\right) d s \\
& \leqslant \frac{1}{\Gamma(\alpha)} \int_{0}^{1} s(1-s)^{\alpha-1} q_{2}(s) g(y(s)) d s \\
& \leqslant \frac{M_{2} r_{2}}{\Gamma(\alpha)} \int_{0}^{1} s(1-s)^{\alpha-1} q_{2}(s) d s \\
& =r_{2}=\|y\|,
\end{aligned}
$$

which yields $\left\|T^{*} y\right\| \leqslant\|y\|$ for any $y \in K \cap \partial \Omega_{r_{2}}$.

Therefore, $T^{*}$ has a fixed point $y_{1}$ in $\bar{\Omega}_{r_{2}} \backslash \Omega_{\rho_{6}}$, and a fixed point $y_{2}$ in $\bar{\Omega}_{\rho_{7}} \backslash \Omega_{\mathrm{r}_{2}}$. Thus, system (1.1) has at least two positive solutions $u_{1}=t^{\alpha-2} y_{1}, u_{2}=t^{\alpha-2} y_{2}, t \in[0,1]$. The proof is completed.

Theorem 3.8. Assume that

$$
\text { (P7) } g^{0}=\alpha_{1} \in\left[0, M_{2}\right) \text { and }\left(\text { P8) } g_{\infty}=\beta_{1} \in\left(\frac{16}{3(\alpha-1)} M_{1}, \infty\right)\right.
$$

hold, where $M_{1}, M_{2}$ are defined in Theorem 3.5. Then system (1.1) has at least one positive solution.

Proof. By (P7), for $\varepsilon=M_{2}-\alpha_{1}>0$, there exists a sufficiently small $\hat{r}_{2}>0$ such that

$$
\max \frac{g(y)}{y}<\alpha_{1}+\varepsilon=M_{2}, \quad \text { for } \quad y \leqslant \hat{r}_{2},
$$

which yields $g(y)<M_{2} y \leqslant M_{2} \hat{r}_{2}$. Hence, the condition (P6) is satisfied.

By (P8), for $\varepsilon=\beta_{1}-\frac{16}{3(\alpha-1)} M_{1}>0$, there exists a sufficiently large $r_{1}>0$, such that

$$
\min \frac{g(y)}{y}>\beta_{1}-\varepsilon=\frac{16}{3(\alpha-1)} M_{1}, \quad \text { for } \quad y \geqslant \frac{3(\alpha-1)}{16} r_{1}
$$

Thus, when $\frac{3(\alpha-1)}{16} r_{1} \leqslant y \leqslant r_{1}$, we have

$$
g(y)>\frac{16}{3(\alpha-1)} M_{1} y \geqslant \frac{16}{3(\alpha-1)} M_{1} \cdot \frac{3(\alpha-1)}{16} r_{1}=M_{1} r_{1}
$$

which implies the condition (P5) hold. By Theorem 3.5, we complete the proof.

Theorem 3.9. Assume that

$$
\text { (P9) } g_{0}=\alpha_{2} \in\left(\frac{16}{3(\alpha-1)} M_{1}, \infty\right) \text { and }(\mathrm{P} 10) \quad g^{\infty}=\beta_{2} \in\left[0, M_{2}\right)
$$

hold, where $M_{1}, M_{2}$ are defined in Theorem 3.5. Then system (1.1) has at least one positive solution.

Proof. By (P9), for $\varepsilon=\alpha_{2}-\frac{16}{3(\alpha-1)} M_{1}>0$, there exists a sufficiently small $\hat{r}_{1}>0$ such that

$$
\min \frac{g(y)}{y}>\alpha_{2}-\varepsilon=\frac{16}{3(\alpha-1)} M_{1}, \quad \text { for } \quad y \leqslant \hat{r}_{1} .
$$

Thus, when $\frac{3(\alpha-1)}{16} \hat{r}_{1} \leqslant y \leqslant \hat{r}_{1}$, one has,

$$
g(y)>\frac{16}{3(\alpha-1)} M_{1} y \geqslant M_{1} \hat{r}_{1}
$$


which yields the condition (P5) holds. In view of (P10), for $\varepsilon=M_{2}-\beta_{2}>0$, there exists a sufficiently large $r>0$ such that

$$
\max \frac{g(y)}{y}<\beta_{2}+\varepsilon=M_{2}, \quad \text { for } y>r .
$$

In the following, we will show that (P6) holds and the discussion is divided into two cases.

Case one: Suppose that $g(y)$ is unbounded, then exists $g^{*} \in C([0,+\infty),[0,+\infty))$ such that $g(y) \leqslant g^{*}\left(y^{*}\right)$ for $y \leqslant y^{*}$ and when $y^{*}>r, \max \frac{g^{*}\left(y^{*}\right)}{y^{*}}<M_{2}$. If we choose $y^{*}=r_{2}>r$, we will get

$$
g(y) \leqslant g^{*}\left(y^{*}\right)<M_{2} y^{*}=M_{2} r_{2} \text { for } y \leqslant y^{*}=r_{2},
$$

which yields the condition (P6) holds.

Case two: Suppose that $g(y)$ is bounded, there exists an $M>0$, such that for any $y$, we have $g(y) \leqslant M$. In this case, taking sufficiently large $r_{2} \geqslant \frac{M}{M_{2}}$, then $g(y) \leqslant M \leqslant M_{2} r_{2}$ for $0<y<r_{2}$, which implies the condition (P6) holds.

Therefore, by Theorem 3.5 we complete the proof.

Theorem 3.10. Assume that (P6), (P8), and (P9) hold. Then system (1.1) has at least two positive solutions.

Proof. From (P8) and the proof of Theorem 3.8, we know that there exists a sufficiently large $r_{1}>r_{2}$ such that

$$
g(y)>M_{1} r_{1} \text {, for } \frac{3(\alpha-1)}{16} r_{1} \leqslant y \leqslant r_{1} .
$$

In view of (P9) and the proof of Theorem 3.9, we see that there exists a sufficiently small $r_{1}^{*} \in\left(0, r_{2}\right)$ such that

$$
g(y)>M_{1} r_{1}^{*}, \text { for } \frac{3(\alpha-1)}{16} r_{1}^{*} \leqslant y \leqslant r_{1}^{*}
$$

Noting that (P6) is valid, then from the proof of Theorem 3.5, we know $\mathrm{T}^{*}$ has at least two fixed points. Thus, system (1.1) has at least two positive solutions. The proof is completed.

Theorem 3.11. Assume that (P5), (P7), and (P10) hold. Then system (1.1) has at least two positive solutions.

Proof. From (P7) and the proof of Theorem 3.8, we know that there exists a sufficiently small $r_{2} \in\left(0, r_{1}\right)$ such that

$$
g(y) \leqslant M_{2} r_{2}, \text { for } 0<y \leqslant r_{2} .
$$

In view of (P10) and the proof of Theorem 3.9, we see that there exists a sufficiently large $r_{2}^{*}>r_{1}$ such that

$$
g(y) \leqslant M_{2} r_{2}^{*}, \text { for } 0<y \leqslant r_{2}^{*} .
$$

Noting that (P5) is also satisfied, then from the proof of Theorem 3.5, we know $\mathrm{T}^{*}$ has at least two fixed points. Thus, system (1.1) has at least two positive solutions. The proof is completed.

Theorem 3.12. Assume that (P1) and (P10) hold. Then system (1.1) has at least one positive solution.

Proof. In view of (P1), we know from the proof of Theorem 3.3 that, for any $y \in K \cap \partial \Omega_{\rho_{0}},\left\|T^{*} y\right\| \geqslant\|y\|$.

By (P10), it follows from the proof of Theorem 3.9, there exists a sufficiently large $r_{2}>\rho_{0}$ such that $g(y) \leqslant M_{2} r_{2}$ for $0<y \leqslant r_{2}$ and $\left\|T^{*} y\right\| \leqslant\|y\|$. This completes the proof.

Similar to Theorem 3.12, one immediately has the following consequences.

Theorem 3.13. Assume that (P2) and (P9) hold. Then system (1.1) has at least one positive solution.

Theorem 3.14. Assume that (P3) and (P8) hold. Then system (1.1) has at least one positive solution. 
Theorem 3.15. Assume that (P4) and (P7) hold. Then system (1.1) has at least one positive solution.

Theorem 3.16. Assume that (P1), (P6), and (P8) hold. Then system (1.1) has at least two positive solutions.

Proof. In view of (P1), we know from the proof of Theorem 3.3 that $\left\|T^{*} y\right\| \geqslant\|y\|$ for any $y \in K \cap \partial \Omega_{\rho_{0}}$.

Since (P8), from the proof of Theorem 3.8, there exists a sufficiently large $r_{1}>r_{2}$ such that $g(y) \geqslant M_{1} r_{1}$ for $\frac{3(\alpha-1)}{16} r_{1} \leqslant y \leqslant r_{1}$.

For any $y \in K \cap \partial \Omega_{r_{1}}$, we have

$$
\begin{aligned}
\left\|T^{*} y\right\| & \geqslant\left(T^{*} y\right)\left(\frac{1}{2}\right) \\
& =\int_{0}^{1} G^{*}\left(\frac{1}{2}, s\right) f\left(s, s^{\alpha-2} y(s)\right) d s \\
& \geqslant \frac{\alpha-1}{4 \Gamma(\alpha)} \int_{\frac{1}{4}}^{\frac{3}{4}} s(1-s)^{\alpha-1} q_{1}(s) g(y(s)) d s \\
& \geqslant \frac{M_{1}(\alpha-1)}{4 \Gamma(\alpha)} r_{1} \int_{\frac{1}{4}}^{\frac{3}{4}} s(1-s)^{\alpha-1} q_{1}(s) d s \\
& =r_{1}=\|y\| .
\end{aligned}
$$

Further, from the proof of Theorem 3.5, for any $y \in K \cap \partial \Omega_{r_{2}}$, we have $\left\|T^{*} y\right\| \leqslant\|y\|$. Therefore, system (1.1) has at least two positive solutions. The proof is completed.

The following statements are immediately obtained by applying similar arguments as used in the proof of Theorem 3.16 .

Theorem 3.17. Assume that (P2), (P5), and (P7) hold. Then system (1.1) has at least two positive solutions.

Theorem 3.18. Assume that (P3), (P5), and (P10) hold. Then system (1.1) has at least two positive solutions.

Theorem 3.19. Assume that (P4), (P6), and (P9) hold. Then system (1.1) has at least two positive solutions.

\section{Nonexistence of positive solutions of (1.1)}

In this section, we will explore the nonexistence of positive solutions of system (1.1).

Theorem 4.1. Assume that (P8), (P9), and the following condition hold

$$
\text { (P11) } \min _{\hat{r}_{1}<y<\frac{3(\alpha-1)}{16} r_{1}} \frac{g(y)}{y} \in\left(\frac{16}{3(\alpha-1)} M_{1}, \infty\right),
$$

where $r_{1}, \hat{r}_{1}$ are defined as the proof of Theorems 3.6 and 3.7, respectively. Then (1.1) has no positive solution.

Proof. From (P8) and the proof of Theorem 3.8, it follows that there exists a sufficiently large $r_{1}>\hat{r}_{1}$ such that $g(y) \geqslant \frac{16}{3(\alpha-1)} M_{1} y$ for $y \geqslant \frac{3(\alpha-1)}{16} r_{1}$.

From (P9), and the proof of Theorem 3.9, it follows that there exists a sufficiently small $\hat{r}_{1}>0$ such that $g(y) \geqslant \frac{16}{3(\alpha-1)} M_{1} y$ for $y \leqslant \hat{r}_{1}$.

By $(P 11)$, we can obtain $g(y) \geqslant \frac{16}{3(\alpha-1)} M_{1} y$ for any $y$.

If $T^{*}$ has a fixed point $y$, then

$$
\|y\|=\left\|T^{*} y\right\| \geqslant\left(T^{*} y\right)\left(\frac{1}{2}\right)=\int_{0}^{1} G^{*}\left(\frac{1}{2}, s\right) f\left(s, s^{\alpha-2} y(s)\right) d s
$$




$$
\begin{aligned}
& >\frac{\alpha-1}{4 \Gamma(\alpha)} \int_{\frac{1}{4}}^{\frac{3}{4}} s(1-s)^{\alpha-1} q_{1}(s) g(y(s)) d s \\
& \geqslant \frac{\alpha-1}{4 \Gamma(\alpha)} \cdot \frac{16}{3(\alpha-1)} M_{1} \int_{\frac{1}{4}}^{\frac{3}{4}} s(1-s)^{\alpha-1} q_{1}(s) y(s) d s \\
& \geqslant \frac{\alpha-1}{4 \Gamma(\alpha)} \cdot \frac{16}{3(\alpha-1)} M_{1} \cdot \frac{3}{16}(\alpha-1)\|y\| \int_{\frac{1}{4}}^{\frac{3}{4}} s(1-s)^{\alpha-1} q_{1}(s) d s \\
& =\|y\|,
\end{aligned}
$$

which is a contradiction. The claim is valid.

The next consequence is presented below whose proof is similar to that of Theorem 4.1, and therefore is omitted.

Theorem 4.2. Assume that(P7), (P10), and the following condition hold

$$
\text { (P12) } \max _{\hat{r}_{2}<y<r_{2}} \frac{g(y)}{y} \in\left[0, M_{2}\right),
$$

where $\hat{r}_{2}, r_{2}$ are defined as the proof of Theorems 3.8 and 3.9, respectively. Then system (1.1) has no positive solution.

\section{References}

[1] M. Al-Akaidi, Fractal speech processing, Cambridge University Press, Cambridge, (2004). 1

[2] A. Babakhani, V. Daftardar-Gejji, Existence of positive solutions of nonlinear fractional differential equations, J. Math. Anal. Appl., 278 (2003), 434-442. 1

[3] Z. B. Bai, H. S. Lü, Positive solutions for boundary value problem of nonlinear fractional differential equation, J. Math. Anal. Appl., 311 (2005), 495-505. 1, 2

[4] L. Bi, M. Bohner, M. Fan, Periodic solutions of functional dynamic equations with infinite delay, Nonlinear Anal., 68 (2008), 1226-1245. 1

[5] D. Delbosco, L. Rodino, Existence and uniqueness for a nonlinear fractional differential equation, J. Math. Anal. Appl., 204 (1996), 609-625. 1

[6] D.-Q. Jiang, C.-J. Yuan, The positive properties of the Green function for Dirichlet-type boundary value problems of nonlinear fractional differential equations and its application, Nonlinear Anal., 72 (2010), 710-719. 1, 2

[7] M. A. Krasnoselskiǔ, Positive solutions of operator equations, Translated from the Russian by Richard E. Flaherty; edited by Leo F. Boron P., Noordhoff Ltd. Groningen, (1964). 2

[8] K. S. Miller, B. Ross, An introduction to the fractional calculus and fractional differential equations, A Wiley-Interscience Publication, John Wiley \& Sons, Inc., New York, (1993). 1

[9] K. B. Oldham, J. Spanier, The fractional calculus, Theory and applications of differentiation and integration to arbitrary order, With an annotated chronological bibliography by Bertram Ross, Mathematics in Science and Engineering, Academic Press [A subsidiary of Harcourt Brace Jovanovich, Publishers], New York-London, (1974). 1

[10] I. Podlubny, Fractional differential equations, An introduction to fractional derivatives, fractional differential equations, to methods of their solution and some of their applications, Mathematics in Science and Engineering, Academic Press, Inc., San Diego, CA, (1999). 1

[11] D. Ye, M. Fan, H.-Y. Wang, Periodic solutions for scalar functional differential equations, Nonlinear Anal., 62 (2005), 1157-1181. 1

[12] Z.-J. Zeng, Existence and multiplicity of positive periodic solutions for a class of higher-dimension functional differential equations with impulses, Comput. Math. Appl., 58 (2009), 1911-1920. 1 